\title{
Dyakonons in hyperbolic metamaterials
}

\author{
C. J. Zapata-Rodríguez, ${ }^{* 1}$ J. J. Miret, ${ }^{2}$ S. Vuković,${ }^{3,4}$ and Z. Jakšić ${ }^{3}$ \\ ${ }^{I}$ Department of Optics, University of Valencia, Dr. Moliner 50, 46100 Burjassot, Spain, \\ ${ }^{2}$ Department of Optics, Pharmacology and Anatomy, University of Alicante, P.O. Box 99, Alicante, Spain, \\ ${ }^{3}$ Center of Microelectronic Technologies and Single Crystals, Institute of Chemistry, Technology and Metallurgy, \\ University of Belgrade, Njegoseva 12, 11000 Belgrade, Serbia, \\ ${ }^{4}$ Texas A \& M University at Qatar, P.O. Box 23874, Doha, Qatar
}

Received March 26, 2013; accepted May 14, 2013; published June 30, 2013

\begin{abstract}
We have analyzed surface-wave propagation that takes place at the boundary between an isotropic medium and a semi-infinite metal-dielectric periodic medium cut normally to the layers. In the range of frequencies where the periodic medium shows hyperbolic space dispersion, hybridization of surface waves (dyakonons) occurs. At low to moderate frequencies, dyakonons enable tighter confinement near the interface in comparison with pure SPPs. On the other hand, a distinct regime governs the dispersion of dyakonons at higher frequencies.
\end{abstract}

Multilayered metal-dielectric (MD) materials can be considered the simplest metamaterials, but also highly versatile, which may be able to sustain bulk high-frequency plasmonic modes. If such a metamaterial is cut perpendicularly to the layers, it can also sustain electromagnetic surface waves. This property has been exploited to excite ultra-confined surfaces waves (also known as spoof plasmons) in the terahertz, which is a regime where conventional surface plasmons cannot exist [1]. For infrared and visible wavelengths, the MD compounds may behave as plasmonic crystals enabling a simplified description of the medium by using the long-wavelength approximation, which explicitly involves the homogenization of the structured metamaterial [2]. Under certain conditions, the permittivity that is given as a second-rank uniaxial tensor may contain elements of the opposite sign, leading to hyperbolic metamaterials [3, 4]. In this context, Jacob et al. [5] showed for the first time the existence of Dyakonovlike surface waves [6] when considering anisotropic media with indefinite permittivity. However, the authors provided only a qualitative and elusive study of hyperbolic dyakonons.

Here, we retake the task and perform a thorough analysis of dyakonons taking place in semi-infinite MD lattices showing hyperbolic dispersion. Our approach puts emphasis on the effective-medium approximation (EMA). Different regimes are found and analyzed thoroughly. Finally, the main conclusions are outlined.

${ }^{*}$ E-mail: carlos.zapata@uv.es

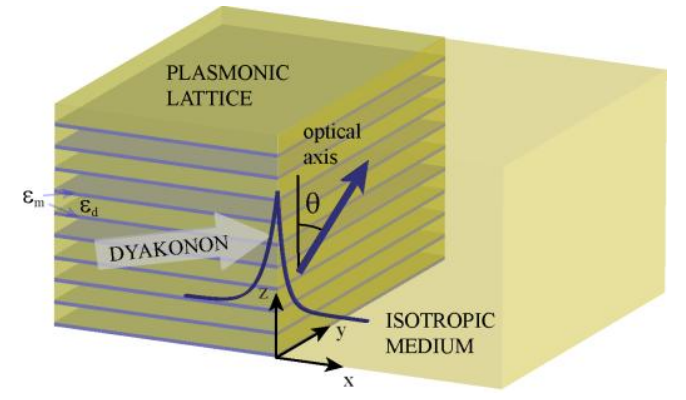

Fig. 1. Scheme of the semi-infinite lattice $(x<0)$, composed of a Drude metal of permittivity $\varepsilon_{\mathrm{m}}$ and a dielectric with $\varepsilon_{\mathrm{d}}=2.25$, together

with an isotropic medium (simulations consider air in $x>0$ ).

The photonic system under consideration is shown in Fig. 1. The periodic bilayered structure is made of two materials alternatively stacked along the $z$ axis. This metamaterial fills the semi-space: $x<0$. Specifically, a transparent material of dielectric constant $\varepsilon_{d}$ and slab width $w_{d}$ is followed by a metallic layer with its corresponding parameters $\varepsilon_{m}$ and $w_{m}$. In our numerical simulations we set $\varepsilon_{m}=1-\omega^{-2}$, which applies to a Drude metal (frequencies in units of the plasma frequency $\omega_{p}$ ). For the purpose of this Letter we will neglect losses, leaving their detailed consideration for the future publication. Finally, we set an isotropic material of dielectric constant $\varepsilon$ filling the space $x>0$.

By employing EMA we modeled the form anisotropy of the described plasmonic device [2]. The validity of EMA is usually related to the assumption that the period $\Lambda=w_{m}+w_{d}$ is much shorter than the wavelength. However, the inclusion of nanostructured metallic elements deserves refinement in the EMA because of the fact that the width of metallic layers can be higher than the skin depth of noble metals, which is deeply subwavelength. Material homogenization requires the metallic layers to be of the size of a few nanometers [8, 9]. In this case, the plasmonic lattice behaves like a uniaxial crystal whose optical axis is normal to the layers (the $z$ axis in Fig. 1). The dyadic relative permittivity is set as 
$\overline{\bar{\varepsilon}}=\varepsilon_{\perp}(\mathbf{x} \mathbf{x}+\mathbf{y y})+\varepsilon_{\|} \mathbf{z}$, where $\varepsilon_{\|}$and $\varepsilon_{\perp}$ are evaluated analytically using [2]

$$
\left[\varepsilon_{\perp}, \varepsilon_{\|}^{-1}\right]=(1-f)\left[\varepsilon_{d}, \varepsilon_{d}^{-1}\right]+f\left[\varepsilon_{m}, \varepsilon_{m}^{-1}\right],
$$

where $f=w_{m} /\left(w_{d}+w_{m}\right)$ stands for the metallic filling factor in the unit cell. Therefore, the engineered anisotropy of the 1D lattice is modulated by the filling factor of the metal, but also by its strong dispersive character.

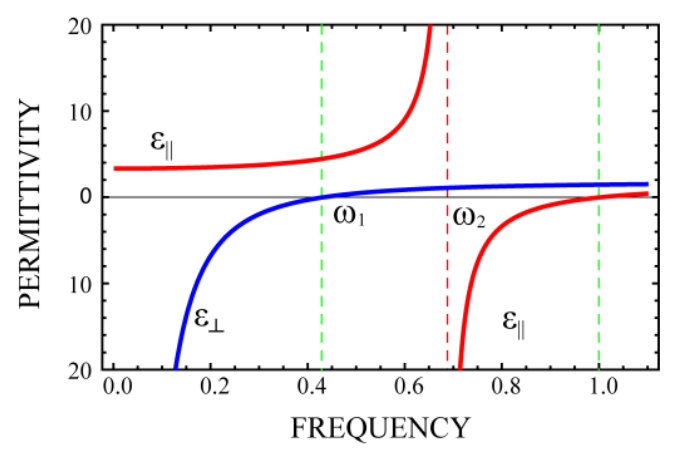

Fig. 2. Permittivities $\varepsilon_{\|}$and $\varepsilon_{\perp}$ as a function of normalized frequency $\omega$ for the MD lattice of Fig. 1 assuming $f=0.33$.

Dispersion in metals determines actively the characteristics of plasmonic-crystal anisotropy [10]. In Fig. 2 we represent permittivities $\varepsilon_{\|}$and $\varepsilon_{\perp}$ for a wide range of frequencies, when $f=0.33$. In the low-frequency range, $\omega<<1$, we obtain that $\varepsilon_{\perp} \approx f \varepsilon_{m}<0$ and $0<\varepsilon_{\|}$ $\approx \varepsilon_{d} /(1-f)$. The plasmonic arrangement behaves essentially as a metal and therefore only evanescent $\mathrm{TE}^{z}$ waves ( $E_{z}=0$ ) can exist. On the other hand, the dispersion of bulk $\mathrm{TM}^{z}$ waves $\left(B_{z}=0\right)$ follows a one-sheet hyperboloid

$$
\frac{k_{x}^{2}+k_{y}^{2}}{\varepsilon_{\|}}+\frac{k_{z}^{2}}{\varepsilon_{\perp}}=\omega^{2},
$$

where spatial frequencies are normalized to $k_{p}=\omega_{p} / c$. The hyperbolic dispersion exists up to a frequency $\omega_{1}=\tilde{\omega}\left(0, \varepsilon_{d}, f\right)$ for which $\varepsilon_{\perp}=0$, being

$$
\tilde{\omega}\left(\varepsilon, \varepsilon_{d}, f\right)=\left[1+\varepsilon / f+\varepsilon_{d}(1-f) / f\right]^{-1 / 2} .
$$

In our numerical example it occurs at $\omega_{1}=0.426$. For slightly higher frequencies, Eq. (2) represents an ellipsoid with a major semi-axis $\omega \sqrt{\varepsilon_{\|}}$. However, note that such a value diverges at a frequency $\omega_{2}=\tilde{\omega}\left(0, \varepsilon_{d}, 1-f\right)$. Here $\omega_{2}=0.686$. Note that $\omega_{1}<\omega_{2}$ only if $f<1 / 2$. Finally, Eq. (2) represents a hyperboloid of two sheets in the range $\omega_{2}<\omega<1$. The frequency in the upper limit is established by the condition $\varepsilon_{\|}=0$, or equivalently $\varepsilon_{m}=0$, occurring at the plasma frequency.

We seek the solutions of the Maxwell's equations in the form of surface waves that can propagate at the boundary of the MD lattice. Therefore, we expect to find localized solutions near $x=0$ with amplitudes decaying as $|x| \rightarrow \infty$. Since we treat the plasmonic lattice as a uniaxial crystal, we may establish analytically the diffraction equation that gives the in-plane wave vector $\mathbf{k}_{D}=\left\lfloor 0, k_{y}, k_{z}\right\rfloor$ of the surface wave. For that purpose we follow Dyakonov [6] by considering the modal treatment of our problem.

In the isotropic medium we include $\mathrm{TE}^{x}$ and $\mathrm{TM}^{x}$ waves. All these fields are evanescent in the isotropic medium, proportional to $\exp (-\kappa x)$, where $\kappa=\tilde{\kappa}(\varepsilon, \varepsilon)$ and

$$
\tilde{\kappa}\left(\varepsilon_{1}, \varepsilon_{2}\right)=\sqrt{k_{y}^{2}+k_{z}^{2} \varepsilon_{1} / \varepsilon_{2}-\varepsilon_{1} \omega^{2}},
$$

in units of $k_{p}$. The time-harmonic modal electric field may be written in the complete form as $\mathbf{E}(\mathbf{r}, t)$ $=\mathbf{E}(x) \exp \left(i \mathbf{k}_{D} \mathbf{r}-i \omega t\right)$. For $x>0$, the $\mathrm{TE}^{x}$ wave may be set as $\mathbf{E}(x)=A_{T E} \mathbf{a} \exp (-\kappa x)$, where $\mathbf{a}=\left\lfloor 0, k_{z},-k_{y}\right\rfloor$. Here time- and space-coordinates are normalized to the inverse of $\omega_{p}$ and $k_{p}$, respectively. Note that the polarization vector a lies on the plane $x=0$, but it is perpendicular to the propagation direction of the dyakonon, $\mathbf{k}_{D}$. The magnetic field of the $\mathrm{TM}^{x}$ mode is conveniently written using $\mathbf{B}(x)=(\varepsilon \omega / c) A_{T M} \mathbf{a} \exp (-\kappa x)$. Both $A_{T E}$ and $A_{T M}$ are complex-valued amplitudes.

On the other side of the boundary, the ordinary $(o-)$ and extraordinary $(e-)$ waves in the effective uniaxial medium also decay exponentially with rates given by $\kappa_{o}=\tilde{\kappa}\left(\varepsilon_{\perp}, \varepsilon_{\perp}\right)$ and $\kappa_{e}=\tilde{\kappa}\left(\varepsilon_{\|}, \varepsilon_{\perp}\right)$, respectively. Specifically, the dependence along the $x$ direction of the $o$ wave electric field in $x<0$ may be written as $\mathbf{E}(x)=A_{o} \mathbf{b}_{o} \exp \left(\kappa_{o} x\right)$. The part of the magnetic field which provides its variation normally to the isotropicuniaxial interface is set now as $\mathbf{B}(x)=A_{e} \varepsilon_{\perp} \omega / c$ $\times \mathbf{b}_{\mathbf{e}} \exp \left(\kappa_{e} x\right)$ for the $e$-wave. Here $A_{o}$ and $A_{e}$ stand for amplitudes of the $o$ - and $e$-waves, respectively. Also we define the vectors $\mathbf{b}_{o, e}=\left[-k_{y},-i \kappa_{o, e}, 0\right]$, which are perpendicular to the optical axis. It is clear that $o$-wave corresponds to a $\mathrm{TE}^{z}$ mode and the $e$-wave essentially is $\mathrm{TM}^{z}$.

Next we apply the standard electromagnetic field boundary conditions at $x=0$, that is, continuity of the $y$ and z-components of the fields $\mathbf{E}$ and $\mathbf{B}$ at the planar interface. This problem may be set in a matrix form as $\overline{\bar{M}} \mathbf{A}=0$, where the vector $\mathbf{A}=\left[A_{T E}, A_{T M}, A_{o}, A_{e}\right]$ includes all the amplitudes. In addition, the matrix

$$
\overline{\bar{M}}=\left[\begin{array}{cccc}
k_{z} & -i k_{y} \kappa & i \kappa_{o} & -k_{y} k_{z} \\
-k_{y} & -i k_{z} \kappa & 0 & \varepsilon_{\perp} \omega^{2}-k_{z}^{2} \\
i k_{y} \kappa & \varepsilon \omega^{2} k_{z} & k_{y} k_{z} & i \varepsilon_{\perp} \omega^{2} \kappa_{e} \\
i k_{z} \kappa & -\varepsilon \omega^{2} k_{y} & k_{z}^{2}-\varepsilon_{\perp} \omega^{2} & 0
\end{array}\right] .
$$

A nontrivial solution for $\mathbf{A}$ may be found if the determinant of $\overline{\bar{M}}$ vanishes. In this way Dyakonov derived the equation giving the spectral map of wave vectors $\mathbf{k}_{D}$ [6] that propagate at the interface between the anisotropic uniaxial dielectric and the isotropic one.

Dyakonons may be found in different regimes, which depends not only on the values characterizing the permittivity of the medium, but also that of the surrounding iso- 
tropic material $\varepsilon$. For illustration, we analyze the case when $\varepsilon<\varepsilon_{d} /(1-f)$ and $f<1 / 2$. Therefore $\varepsilon<\varepsilon_{\|}$in the range $0<\omega<\omega_{1}$, where $\varepsilon_{\perp}<0$. It is easy to see that $\kappa<\kappa_{o}$ and, also $\kappa_{e}<\kappa_{o}$. Moreover, we can find stationary solutions of Dyakonov's equation only if $\varepsilon<-\varepsilon_{\perp}$. This occurs when $0<\omega<\omega_{0}$, where $\omega_{0}=\tilde{\omega}\left(\varepsilon, \varepsilon_{d}, f\right)$; in our numerical simulations $\omega_{0}=0.343$. (a)

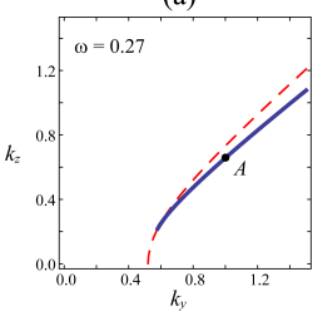

(c)

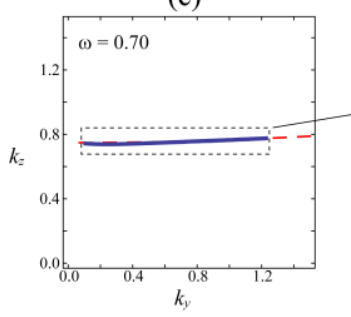

(b)

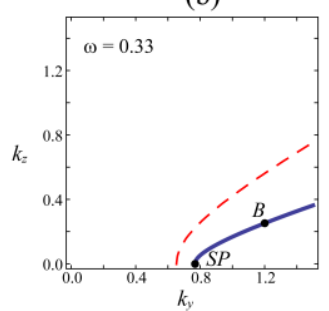

(d)

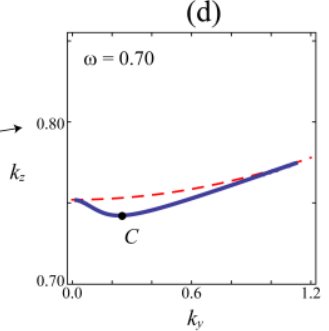

Fig. 3. Solutions of equation $\operatorname{det}(\overline{\bar{M}})=0$, drawn in solid line, considering our hyperbolic metamaterial neighboring an isotropic medium

of permittivity $\varepsilon=1$, at different frequencies: (a) $\omega=0.27$ (b) $\omega=0.33$ (c) $\omega=0.70$. As a reference we also include equation $\kappa_{e}=0$ that is set in dashed line. (d) Dispersion curve as given in (c) but ranged over the region of interest. Points $A, B, C$, and $S P$ are used in Fig. 4.

In Fig. 3(a)-(b) we illustrate the dyakonon dispersion curve for some frequencies $0<\omega<\omega_{0}$. In these two cases, dyakonons curve approaches a hyperbola. In contrast to what is shown in Fig. 3(b), we find a band-gap around $k_{z}=0$ in (a); in general terms it occurs if $\omega<0.324$. In this sense we point out that Dyakonov's solutions at $k_{z}=0$ are constrained to the condition $k_{y} \geq \omega \sqrt{\varepsilon_{\|}}$, which is necessary for $\kappa_{e}$ to exhibit positive values.

Next we consider the high-frequency band $\omega_{2}<\omega<1$ where $\varepsilon_{\|}<0<\varepsilon_{\perp}$. The plot shown in Fig. 3(c) corresponds to this case. The dyakonons curve goes near the curve $\kappa_{e}=0$, as it happens in Fig. 3(a) and (b). However, it crosses the curve $\kappa_{e}=0$ at two different points, where the curve begins and ends. In comparison, the angular range of dyakonons becomes significantly low.

Figure 4 shows the magnetic field for the points $A, B$, and $C$, all highlighted in Fig. 3. Also we include the field at the point $S P$ shown in Fig. 3(b), which corresponds to a surface plasmon ( $B_{x}=0$ ). For the cases $A$ and $B$, the field pattern remains tightly confined near the surface $x=0$ within a few units of $1 / k_{p}$. Such wave localization is even stronger than the confinement of the surface plasmon, appearing at $\omega=0.33$ for $k_{y}=0.766$. This is caused by the large in-plane wavenumber of the dyakonon; note that $k_{z}=0.660$ for the point $A$ and $k_{z}=0.254$ for the point $B$. By contrast, the lowest confinement is produced at point $C: \mathbf{k}_{D}=[0,0.25,0.74]$ at $\omega=0.70$. In this case, the dominant contribution of the extraordinary wave counts against localization near the surface.

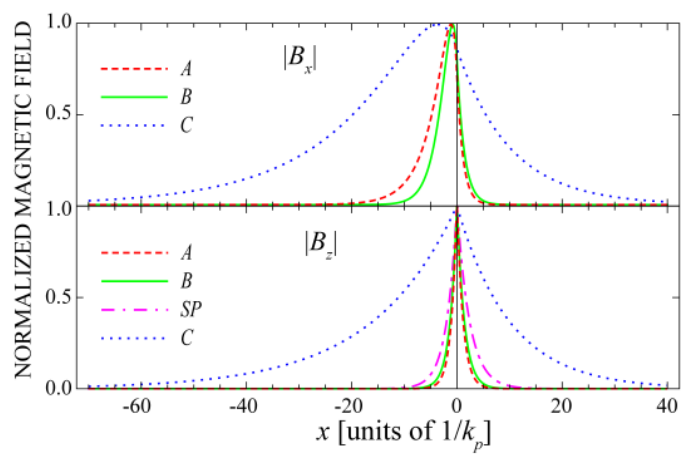

Fig. 4. Variation of magnetic field along the x-axis for the points $A, B$, and $C$ highlighted in Fig. 3. We include the point $S P$ associated with pure $\mathrm{TM}^{x}$ surface waves.

In summary, we have shown that Dyakonov-like surface waves excited in hyperbolic metamaterials enable distinct regimes of propagation. At low and moderate frequencies, dyakonons propagate in the canalization regime, as shown in Ref. 5. However, bandgaps may be found when surface waves are directed parallel to the layers. At high frequencies, severe restrictions arise concerning the existence of a narrow angular range. Finally, we have pointed out that the properties of the resulting bound states change rapidly with the refractive index of the surrounding medium, suggesting potential applications for chemical and biological sensors.

This research was funded by the Spanish Ministry of Economy and Competitiveness (project TEC2011-29120C05-01) and by the Qatar National Research Fund (project NPRP 09-462-1-074). CJZR gratefully acknowledges a financial support from the Generalitat Valenciana (grant BEST/2012/060).

\section{References}

[1] Z. Ruan, M. Qiu, Appl. Phys. Lett. 90, 201906 (2007).

[2] A. Yariv, P. Yeh, J. Opt. Soc. Am. 67, 438 (1977).

[3] D.R. Smith, D. Schurig, Phys. Rev. Lett. 90, 077405 (2003).

[4] I.I. Smolyaninov, E. Hwang, E. Narimanov, Phys. Rev. B 85, 235122 (2012).

[5] Z. Jacob, E.E. Narimanov, Appl. Phys. Lett. 93, 221109 (2008).

[6] M.I. D'yakonov, Sov. Phys. JETP 67, 714 (1988).

[7] I.I. Smolyaninov, Y.J. Hung, C.C. Davis, Science 315, 1699 (2007).

[8] J. Elser, V.A. Podolskiy, I. Salakhutdinov, I. Avrutsky, Appl. Phys. Lett. 90, 191109 (2007).

[9] E. Popov, S. Enoch, Opt. Lett. 32, 3441 (2007).

[10] B. Wood, J.B. Pendry, D.P. Tsai, Phys. Rev. B 74, 115116 (2006). 